\title{
Une médecine plus humaine est-elle plus efficace?
}

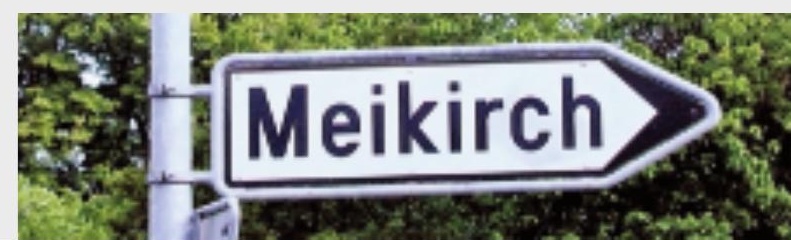

Meikirch? Non, ce terme ne désigne ni un carrosse de luxe ni un appel d'urgence international, encore moins une variante de glandes tarsiennes, même s'il peut prêter à de telles associations d'idées. Jusqu'à présent Meikirch était seulement le nom d'une petite bourgade près de Berne - mais cela pourrait changer. Ce terme deviendra peut-être le symbole du message délivré par un livre paru récemment*, dont l'ambition apparaît clairement sur l'illustration de la couverture: on y voit un panneau indicateur montrant la direction à prendre, en l'occurrence celle de Meikirch.

Le thème traité dans cet ouvrage par les médecins Johannes Bircher et Karl-H. Wehkamp (qui est aussi sociologue et philosophe) est d'actualité et en préoccupe plus d'un. Contre toute attente, les progrès incroyables accomplis au cours des dernières décennies placent la médecine ou plutôt notre système de santé global dans de sérieuses difficultés, les plus visibles étant les limitations économiques auxquelles les médecins sont de plus en plus confrontés. «Rationnement», tel est le nom de la nouvelle épée de Damoclès qui pend au-dessus des décideurs médicaux. La solidité du fil qui la tient en suspens est de plus en plus déterminée par des politiciens et des économistes. Un fossé sépare apparemment leurs exigences de la réalité de la médecine. Un des buts sublimes du TARMED était, par exemple, de valoriser les prestations médicales intellectuelles, à l'exemple des entretiens médecin-patient mais, dans les faits, on observe plutôt le contraire. La fragmentation des sous-disciplines hautement spécialisées continue bravement sa progression, ce qui incite les médias tout public - comme récemment le magazine Facts - à entonner, dans une sorte d'euphorie de fuite en avant, un hymne à la technocratisation totale de la médecine. On doute gentiment que cet avis soit partagé par la majorité des patients et des médecins.

Le problème n'est certes pas le progrès technologique qui a ouvert à la médecine des voies inimaginables il y a peu encore, et aucune personne raisonnable ne peut vouloir sérieusement retourner à l'âge de la pierre. Le problème consiste en la réduction de facto de l'être humain à ce qui peut être saisi et amélioré par la technique selon le principe «Ce que nous ne pouvons pas mesurer n'est pas important». Il est pratique- ment indiscutable qu'une telle conception de l'être humain ne tient pas compte de certaines de ses dimensions intrinsèques essentielles. Ce constant n'a pourtant guère influencé jusqu'à présent la pratique de la médecine (de pointe).

On perçoit des signes indiquant que cette situation pourrait changer car on observe une nouveauté de taille: les voix en provenance de la médecine de pointe se multiplient pour dire que les tendances décrites conduisent à péjorer le résultat. Le style narratif choisi par Bircher et Wehkamp met en exergue les absurdités du système avec une évidence quasi irréfutable et les lecteurs les reconnaissent sans peine. Ces absurdités augmentent les coûts de la médecine, d'où l'exigence paradoxale d'une médecine «plus humaine» ou peut-être mieux adaptée aux besoins de l'être humain, ne fût-ce que dans un but d'efficacité.

Bircher et Wehkamp vont dans cette direction: ils ont intelligemment intitulé leur livre «Das ungenutzte Potential der Medizin» (Le potentiel inutilisé de la médecine). Cette potentialité se trouve aussi au cœur du «modèle Meikirch» qu'ils ont développé (Meikirch est le lieu d'habitation de l'un des deux auteurs). Aux capacités naturelles qui déclinent tout au long de la vie, ils opposent les aptitudes acquises, qui augmentent jusqu'à la fin du séjour terrestre. Ils estiment qu'il faut toujours tenir compte de ces deux aspects dans les examens et constats médicaux et les mettre en lien avec la responsabilité personnelle et les exigences de la vie. Prises isolément, ces affirmations ont une résonnance très théorique mais elles acquièrent de la consistance et deviennent vivantes dans le contexte du livre grâce à la forme narrative choisie, à savoir une fiction sur le cursus de trois jeunes médecins.

Si des propositions telles que le modèle de Meikirch trouvaient un écho favorable dans les centres décisionnels de notre système de santé, il en résulterait effectivement de nombreuses conséquences très intéressantes pour la médecine, conformément au postulat des auteurs. Vues objectivement, leurs réflexions sont une goutte d'eau dans une mer d'opinions et d'idées. Mais qu'en est-il du coup d'aile d'un papillon qui déclencha une tornade de l'autre côté du globe? L'avenir nous le dira.

Bruno Kesseli
2006. 256 pages.

ISBN-13 978-3-907625-31-6. 